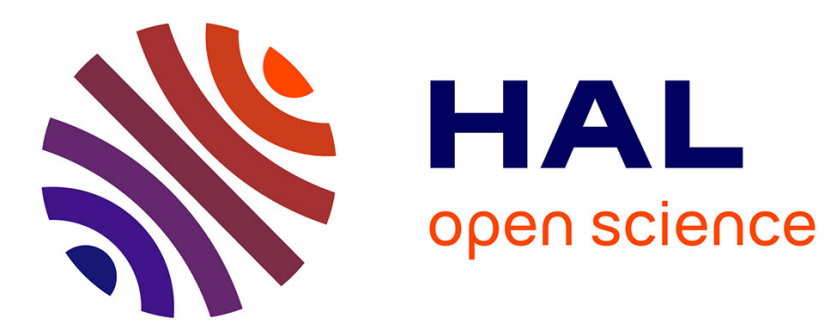

\title{
Tuning of Robust Distant Downstream PI Controllers for an Irrigation Canal Pool: (II) Implementation Issues
}

\author{
X. Litrico, V. Fromion, J.P. Baume
}

\section{To cite this version:}

X. Litrico, V. Fromion, J.P. Baume. Tuning of Robust Distant Downstream PI Controllers for an Irrigation Canal Pool: (II) Implementation Issues. Journal of Irrigation and Drainage Engineering, 2006, 132 (4), p. 369 - p. 379. 10.1061/(ASCE)0733-9437(2006)132:4(369) . hal-00450868

\section{HAL Id: hal-00450868 https://hal.science/hal-00450868}

Submitted on 27 Jan 2010

HAL is a multi-disciplinary open access archive for the deposit and dissemination of scientific research documents, whether they are published or not. The documents may come from teaching and research institutions in France or abroad, or from public or private research centers.
L'archive ouverte pluridisciplinaire HAL, est destinée au dépôt et à la diffusion de documents scientifiques de niveau recherche, publiés ou non, émanant des établissements d'enseignement et de recherche français ou étrangers, des laboratoires publics ou privés. 


\title{
Tuning of Robust Distant Downstream PI Controllers for an Irrigation Canal Pool: (II) Implementation Issues
}

\author{
X. Litrico, V. Fromion ${ }^{\dagger}$ and J.-P. Baume \\ Paper published in the Journal of Irrigation and Drainage Engineering, Vol. 132, No. \\ 4, pp. 369-379, July/August 2006
}

\begin{abstract}
Based on the companion paper results, this paper presents a robust tuning method to tune a PI controller that fulfills the design requirements for a single pool with different hydraulic conditions. The robust PI controller parameters are obtained analytically as function of the physical parameters of the canal pool. Important implementation issues are also considered. Rules are provided for the sampling time selection in order to recover the continuous-time performance. When the sampling time is imposed, it has to be included in the controller design, by modifying the delay of the system. A simple way is proposed to take account of the gate opening as control action variable, instead of the upstream discharge. This robust PI tuning method is evaluated by simulation on a full nonlinear model of two different canal pools for different flow conditions and different implementations: continuous-time control, discrete-time control, using the discharge or the gate opening as control action variable. Simulation results show that the method leads to efficient realistic PI controllers for a canal pool.
\end{abstract}

\section{Introduction}

The development of a systematic method to tune simple and robust controllers is still a challenging issue for irrigation canals. The most widespread controllers in industry are Proportional Integral (PI) controllers. Indeed, when correctly tuned, these simple controllers achieve good performance and robustness. Different tuning methods have already been proposed in the literature for distant downstream PI control of irrigation canals. Existing methods are mainly based on trial-and-error process, or on optimization (see e.g. Deltour and Sanfilippo (1998); Baume et al. (1999); Clemmens and Schuurmans (2004); Clemmens and Wahlin (2004)). These methods are usually based on a nominal model, while the model of the system varies with the hydraulic conditions. It would be desirable to provide a simple way to tune controllers according to a minimum performance, guaranteed for a set of hydraulic conditions. This is the robust performance design problem.

Schuurmans et al. (1999b) appear to be the first to provide a robust tuning method of PI controller for an irrigation canal. They propose a rule to tune a robust PI controller for a canal pool represented by an Integrator Delay model, where the control input is the upstream discharge and the controlled output the downstream water level. The tuning rule provides robust PI parameters for various ID model parameters corresponding to different flow regimes. The designed controller appears to be efficient and robust, but the method lacks some flexibility in the design, since the controller parameters cannot be

\footnotetext{
${ }^{*}$ Researcher, UMR G-EAU, Cemagref, B.P. 5095, 34196 Montpellier Cedex 5, France, Tel: +33 4670463 47. Fax: +334676357 95. e-mail: xavier.litrico@cemagref.fr

${ }^{\dagger}$ Researcher, MIG, Mathematic, Computing Science and Genome, INRA, Domaine de Vilvert, F-78350 Jouy-en-Josas, France. E-mail: vincent.fromion@jouy.inra.fr

${ }^{\ddagger}$ Hydraulic Engineer, UMR G-EAU, Cemagref, B.P. 5095, 34196 Montpellier Cedex 5, France. e-mail: jean-pierre.baume@cemagref.fr
} 
changed according to performance or robustness criteria. The next step would be to give to the designer a systematic tool in order to design a robust PI controller according to a desired minimum level of performance.

Control engineers have developed controller tuning methods based on a frequency domain representation of the system. Contrarily to time-domain methods, it is not easy to relate the frequency domain characteristics to time-domain performance; however, frequency domain methods enable us to explicitly

take account of robustness requirements. In the case of canal pools, we have shown in a companion paper that robustness margins can be directly related to time-domain performance. This property makes controller tuning based on frequency domain representation appealing, because it enables us to tackle two critical requirements concerning the time-domain performance and the robustness.

We developed in the companion paper a method to tune a robust PI controller based on gain and phase margins requirements. This controller was shown to fulfill the design requirements for a given nominal situation corresponding to fixed hydraulic conditions. However, the ultimate goal of a controller is to function under various hydraulic conditions, with at least a given minimal performance. Based on the companion paper results, this paper proposes a robust tuning method that guarantees a specified minimal time-domain performance when the system changes due to discharge or other physical parameters variations.

The paper first considers robust continuous-time control with the upstream discharge as control action variable, because this provides an efficient decoupling of the canal system (Schuurmans, 1997). However, in practice, a canal is controlled with sampled-time controllers, and using gate openings as control action variables. In view of practical implementation, it is important to take account of these issues. The selection of a sampling time period is a crucial point, as it imposes additional constraint on the achievable performance. We provide upper and lower bounds on the sampling time in order to recover the continuous-time performance. However, in the majority of cases, the sampling time period is imposed by hardware (e.g., in order to prevent excessive heating of electrical motors, there are limits on the number of openings of a gate in one hour, etc.). In this case, it is important to take account of the sampling time in the design of the controller.

The fact that the canal is operated using gates as control action variables is also an important issue, since the conversion from discharge to gate opening is not always straightforward, as will be explained below. We show in this paper how to apply the robust PI tuning method for discrete-time control and gate opening as control action variable. The tuning method is illustrated by simulations on a full nonlinear model of two different canal pools taken from the ASCE test cases (Clemmens et al., 1998).

\section{Impact of Model Parameters Variation on the Controlled System}

Let us evaluate the impact of model variation on a controlled canal pool, by designing a PI controller with the method described in the companion paper.

\section{Definition of Variables}

The deviation from upstream discharge is denoted $u_{1}$, the deviation from downstream discharge $u_{2}$, and the deviation from downstream water elevation $y$, while the outlet discharge is denoted $p$. Let $G_{1}(s)$ and $G_{2}(s)$ respectively be the transfer functions from $u_{1}$ to $y$ and from $u_{2}$ to $y$, with $s$ the Laplace variable. The canal pool is therefore represented by:

$$
y=G_{1}(s) u_{1}+G_{2}(s)\left(u_{2}+p\right)
$$

The Integrator Delay model approximation states that the transfer functions $G_{1}(s)$ and $G_{2}(s)$ can be approximated by (see Schuurmans et al. (1999a); Litrico and Fromion (2004)):

$$
\begin{aligned}
& G_{1}(s)=\frac{e^{-\tau_{d} s}}{A_{d} s} \\
& G_{2}(s)=-\frac{1}{A_{d} s}
\end{aligned}
$$

where $A_{d}$ is the equivalent backwater area and $\tau_{d}$ the delay. 


\section{Model Parameters Variation}

To take into account robustness constraints, the ASCE Test Cases explicitly consider different hydraulic conditions by specifying high and low flow conditions and tuned and untuned situations (Clemmens et al., 1998). In the so-called "tuned" situation, the Manning coefficient is equal to 0.014. In the socalled "untuned" situation, the Manning coefficient is equal to 0.018 , and the gate discharge coefficients are $10 \%$ less than in the tuned situation.

Such a variety of hydraulic conditions enable us to evaluate the robustness of a controller to model uncertainties. Indeed, the model parameters vary with the hydraulic conditions. This is illustrated on pool 4 of ASCE Test Canal 1. The pool is $800 \mathrm{~m}$ long, with a bottom slope of 0.002 . The pool has a trapezoidal geometry with bottom width $0.8 \mathrm{~m}$ and side slopes 1.5. The target downstream water level is $0.9 \mathrm{~m}$. For high flow conditions, the discharge is $1.4 \mathrm{~m}^{3} / \mathrm{s}$, while for low flow conditions, it is equal to $0.5 \mathrm{~m}^{3} / \mathrm{s}$. The corresponding ID model parameters are given in table 1 .

[Table 1 about here.]

When the flow decreases, one observes that $\tau_{d}$ and $A_{d}$ increase. Contrarily, in the untuned conditions, the delay increases while the backwater area decreases. In this case, it is not easy to select a nominal model in order to design a controller.

Apart from these model parameters variations due to hydraulic conditions, there are also uncertainties in the gate discharge coefficient, in the gate opening, and also uncertainties in the water levels measurements. Let us compute in this case the relative error in discharge. For submerged conditions, the discharge $Q$ at a gate is classically computed by the following equation:

$$
Q=C_{d} L_{v} W \sqrt{2 g H}
$$

with $C_{d}$ the discharge coefficient, $L_{v}$ the gate width, $W$ the gate opening, $g=9.81 \mathrm{~m}^{2} / \mathrm{s}$ the gravitational acceleration, $H=Y_{1}-Y_{2}$ the hydraulic head, with $Y_{1}$ the upstream gate water level and $Y_{2}$ the downstream gate water level.

The relative error in discharge is classically computed as:

$$
\frac{\delta Q}{Q}=\frac{\delta C_{d}}{C_{d}}+\frac{\delta L_{v}}{L_{v}}+\frac{\delta W}{W}+\frac{1}{2} \frac{\delta H}{H}
$$

Therefore, one may compute the relative discharge uncertainty related to the other relative uncertainties. For example, for ASCE test canal one, the uncertainty in the discharge coefficient is $10 \%$, which means $\delta C_{d} / C_{d}=0.1$. The minimum gate movement of $0.5 \%$ implies a relative uncertainty on $\delta W / W=0.005$. Due to measurement uncertainties, one may also add an uncertainty of $0.5 \%$ on the gate width $\left(\frac{\delta L_{v}}{L_{v}}=\right.$ $0.005)$ and of $2 \%$ on the hydraulic head $(\delta H / H=0.02)$. Finally, the relative uncertainty in $Q$ is:

$$
\frac{\delta Q}{Q}=0.1+0.005+0.005+\frac{0.02}{2}=0.12
$$

Since the uncertainty on the input discharge is $12 \%$, we need to specify a minimum gain margin of $\delta g=1.12$ (i.e., $\Delta G_{\min }=1 \mathrm{~dB}$ ). This is the minimum margin that ensures stability with respect to actuator uncertainties. This is a true stability margin, which provides a strict lower bound to design a stable controller. However, it is not advisable to implement a controller with a gain margin of 1 $\mathrm{dB}$. Indeed, with such a small gain margin, the bandwidth is very large, which makes the controlled system very sensitive to sensor noise, and the control action variable very aggressive. Such a small margin also leads to high overshoot in response to a step reference input. Actually, as shown in the companion paper, the gain margin is strongly related to the performance of the closed-loop system. For irrigation canal control, most of the necessary gain margin is not related to the robustness, but is imposed by performance requirements. Therefore, a minimum performance gain margin of about 4-6 $\mathrm{dB}$ is advisable, that should be added to the minimum gain margin associated to actuator uncertainties. Due to the same reason, it is also advisable to guarantee a minimum phase margin of $\Delta \Phi_{\min }=30^{\circ}$ to have a good time-domain behavior. This also ensures good robustness with respect to other dynamic uncertainties such as delay uncertainties which are measured by the delay margin. 


\section{Nominal PI Tuning for Pool 4 of ASCE Test Canal 1}

To illustrate the interest of a robust performance tuning, let us design a PI controller for pool 4 of ASCE Test canal one using the method proposed in the companion paper, and examine how the time-domain performance degrades when changing from low to high flow and from tuned to untuned situations.

The PI controller is designed to achieve a specified performance at low flow for tuned situation. The reference perturbation discharge is $Q_{p}=0.2 \mathrm{~m}^{3} / \mathrm{s}$. The desired maximum error is $0.2 \mathrm{~m}$ and the settling time $t_{10}=3400 \mathrm{~s}$. In this case, the reference water level deviation is $y_{r}=Q_{p} \times \tau_{d} / A_{d}=$ $0.2 \times 308 / 775=0.08 \mathrm{~m}$. The dimensionless error corresponding to the desired maximum error of $0.2 \mathrm{~m}$ is $e_{\max }^{*}=2.5$. The PI controller design is done following the results of the companion paper, which are summarized in appendix I and II. With table 7, this leads to a gain margin of $13 \mathrm{~dB}$. The dimensionless crossover frequency is given by Eq. (32), $\omega_{c}^{*}=0.352$. Then, the dimensionless time is equal to $t_{10}^{*}=3400 / 308=11$. By linear interpolation from table 8 , this leads to $\Delta \Phi / \Delta \Phi_{\max }=0.5$. Since according to Eq. (33) $\Delta \Phi_{\max }=70^{\circ}$, this leads to $\Delta \Phi=35^{\circ}$. The dimensionless controller parameters are then obtained with the Eqs. (34) and (35): $T_{i}^{*}=4.07$ and $k_{p}^{*}=0.288$. The dimensional values are obtained following (36) and (37): $T_{i}=4.07 \times 308=1254 \mathrm{~s}$ and $k_{p}=0.288 \times 775 / 308=0.725$ $\mathrm{m}^{2} / \mathrm{s}$.

Let us examine the way this controller behaves when applied to the ID model of the canal pool for different hydraulic conditions.

The time-domain simulations show that the design requirements are not fulfilled for all hydraulic conditions: at high flow, the maximum error constraint is violated (see Fig. 1).

[Figure 1 about here.]

With the results of the companion paper, we are therefore able to compute the coefficients of a PI controller that satisfies the design requirements for a given integrator delay model. However, the practical problem is that we search a unique controller for a family of systems (e.g., a canal pool operating at different discharges). It would be possible to proceed by trial and error with the nominal tuning method, in order to obtain a required level of performance. However, as is demonstrated below, it is possible to derive a direct method to tune a PI controller for robust performance (i.e., a guaranteed minimum time-domain performance for all considered hydraulic conditions).

\section{Robust Distant Downstream PI Tuning}

\section{Impact of Model Parameters Variation on Robustness Margins}

The variations of discharge or other parameters affect the gain and the delay of the model. They will therefore modify the gain and phase margins of a PI controller controlling the system. These gain and phase margins variations can be quantified.

To this end, let us study the way gain and phase margins of a given PI controller vary with the parameters of the system: the delay and the integrator gain.

Let $\tilde{G}_{1}(s)=e^{-\tilde{\tau}_{d} s} /\left(\tilde{A}_{d} s\right)$ denote the modified system model with modified parameters denoted with a . The PI controller is denoted $K_{1}(s)$.

Let us express the modified system $\tilde{G}_{1}(s)$ in terms of dimensionless variables, to obtain:

$$
\tilde{G}_{1}(s) K_{1}(s)=G_{1}^{*}\left(\tilde{s}^{*}\right) \tilde{K}_{1}^{*}\left(\tilde{s}^{*}\right)
$$

where $\tilde{s}^{*}=\tilde{\tau}_{d} s$ and $\tilde{K}_{1}^{*}\left(\tilde{s}^{*}\right)$ denotes the dimensionless modified controller, with

$$
\begin{aligned}
\tilde{k}_{p}^{*} & =k_{p}^{*} \frac{\tilde{\tau}_{d}}{\tilde{A}_{d}} \frac{A_{d}}{\tau_{d}} \\
\tilde{T}_{i}^{*} & =T_{i}^{*} \frac{\tau_{d}}{\tilde{\tau}_{d}}
\end{aligned}
$$

In this way, the model parameters variations are transformed into controller parameters variations, which enable us to keep the dimensionless equations to study the controlled system. 
Eqs. (32) and (34) in Appendix I enable us to express the modified gain and phase margins as functions of the modified crossover frequency $\tilde{\omega}_{c}^{*}$ :

$$
\begin{aligned}
\Delta \tilde{G}^{*} & =20 \log _{10}\left(\frac{\tilde{\omega}_{180}^{*}}{\tilde{\omega}_{c}^{*}}\right) \\
\Delta \tilde{\Phi}^{*} & =\frac{180}{\pi}\left(\arctan \left(\tilde{T}_{i}^{*} \tilde{\omega}_{c}^{*}\right)-\tilde{\omega}_{c}^{*}\right)
\end{aligned}
$$

and $\tilde{\omega}_{180}^{*}=\pi / 2$.

According to Eq. (35), the modified dimensionless crossover frequency $\tilde{\omega}_{c}^{*}$ is solution of the following equation:

which leads to:

$$
\tilde{\omega}_{c}^{* 4}-\tilde{k}_{p}^{* 2} \tilde{\omega}_{c}^{* 2}-\frac{\tilde{k}_{p}^{* 2}}{\tilde{T}_{i}^{* 2}}=0
$$

$$
\tilde{\omega}_{c}^{*}=\frac{\tilde{k}_{p}^{*}}{\sqrt{2}} \sqrt{1+\sqrt{1+\frac{4}{\tilde{T}_{i}^{* 2} \tilde{k}_{p}^{* 2}}}}
$$

Therefore the gain and phase margins variations of a fixed PI controller can be directly linked to the system's parameters variations:

$$
\Delta \tilde{G}-\Delta G=20 \log _{10}\left(\frac{\omega_{c}^{*}}{\tilde{\omega}_{c}^{*}}\right)
$$

and

$$
\Delta \tilde{\Phi}-\Delta \Phi=\frac{180}{\pi}\left(\arctan \left(\tilde{T}_{i}^{*} \tilde{\omega}_{c}^{*}\right)-\arctan \left(T_{i}^{*} \omega_{c}^{*}\right)+\omega_{c}^{*}-\tilde{\omega}_{c}^{*}\right)
$$

It is interesting to notice that the gain margin variations are directly linked to the ratio $\omega_{c}^{*} / \tilde{\omega}_{c}^{*}$.

In a first approximation, assuming $\tilde{T}_{i}^{* 2} \tilde{k}_{p}^{* 2} \gg 4$ in Eq. (11), the dimensionless crossover frequency is close to the dimensionless proportional gain of the controller. Then, the gain margin variations are linked to the ratio $k_{p}^{*} / \tilde{k}_{p}^{*}$, since:

$$
\Delta \tilde{G} \approx \Delta G+20 \log _{10}\left(\frac{\tau_{d}}{A_{d}} \frac{\tilde{A}_{d}}{\tilde{\tau}_{d}}\right)
$$

Therefore, one way to guarantee a minimal gain margin is to choose as nominal model the model with the largest $\tau_{d} / A_{d}$ ratio. Indeed, in this case the ratio $\left(\tau_{d} \tilde{A}_{d}\right) /\left(A_{d} \tilde{\tau}_{d}\right)$ becomes larger than 1 for any modified parameters, and according to Eq. (14) the modified gain margin is larger than the nominal one.

Indeed, let us apply these results to evaluate the robustness margins of the robust PI tuning method of Schuurmans et al. (1999b). Schuurmans et al. (1999b) propose the following tuning rules for a sloping reach:

$$
k_{p}=\frac{\min \left\{A_{d}\right\}}{3 \max \left\{\tau_{d}\right\}} \text { and } T_{i}=6 \max \left\{\tau_{d}\right\}
$$

Let us compute the robustness margins of this controller. Assuming that $\min \left\{A_{d}\right\} / \max \left\{\tau_{d}\right\}=\min \left\{A_{d} / \tau_{d}\right\}$, one may compute the minimum gain margin and an average phase margin using Eqs. (9-11). With $k_{p}^{*}=0.33$ and $T_{i}^{*}=6$, this leads to $\Delta G=12.7 \mathrm{~dB}$ and $\Delta \Phi=44.5^{\circ}$. These are reasonable margins which lead to a stable PI controller, as attested by the simulations of Schuurmans et al. (1999b). However, this tuning rule does not guarantee a minimum time-domain performance, since the maximum error and the settling time may vary with the hydraulic parameters.

\section{Robust Performance Tuning Method}

The robust performance design problem consists in tuning a unique PI controller such that the following design specifications are fulfilled for a set of discharges and friction coefficients: 
- for a withdrawal of $Q_{p} \mathrm{~m}^{3} / \mathrm{s}$, the controlled water level should not deviate more than $e_{\max } \mathrm{m}$ from the target water depth.

- after such a withdrawal has occurred, the time for the water level to reach its reference at $\pm 10 \%$ should be at most $t_{10}$ seconds.

Let us denote by $i=1, \ldots, N$ the number of models considered, each model corresponding to different discharges, friction coefficients, etc. To each model corresponds a couple $\left(\tau_{d}(i), A_{d}(i)\right)$.

The maximum acceptable dimensionless error is given for each model by:

$$
e_{\max }^{*}(i)=\frac{A_{d}(i)}{\tau_{d}(i)} \frac{e_{\max }}{Q_{p}}
$$

In order to fulfill the first design specification, one needs to design a controller which gives at most the lowest dimensionless error:

$$
e_{\max }^{*}\left(i_{M}\right)=\min _{i}\left\{e_{\max }^{*}(i)\right\}=\min \left\{\frac{A_{d}}{\tau_{d}}\right\} \frac{e_{\max }}{Q_{p}}
$$

Using table 7 in Appendix II, this gives the gain margin $\Delta G_{M}$ such that the first design specification is fulfilled. If $\Delta G_{M}$ is too small, one needs to increase $e_{\max }$. Else, the corresponding crossover frequency $\omega_{c M}$ is given by Eq. (32):

$$
\omega_{c M}^{*}=\frac{\pi}{2} 10^{\frac{-\Delta G_{M}}{20}}
$$

Since $k_{p}^{*} \approx \omega_{c M}^{*}$, this provides a first approximation of the proportional gain of the controller:

$$
k_{p} \approx \omega_{c M}^{*} \min \left\{\frac{A_{d}}{\tau_{d}}\right\}
$$

With this proportional gain, the dimensionless crossover frequencies can be approximated for each model by:

$$
\omega_{c}^{*}(i)=k_{p} \frac{\tau_{d}(i)}{A_{d}(i)}
$$

Using Eq. (32), the corresponding effective gain margins are given by:

$$
\Delta G(i)=20 \log _{10}\left(\frac{\pi}{2 \omega_{c}^{*}(i)}\right)
$$

The second design specification is transformed into a dimensionless form for each model:

$$
t_{10}^{*}(i)=\frac{t_{10}}{\tau_{d}(i)}
$$

Using Eqs. (21) and (22) with table 8 in Appendix II and Eq. (33), one may compute for each model the phase margin $\Delta \Phi(i)$ such that the second design specification is fulfilled. In order to fulfill this second design requirement, the controller should have a phase margin lower or equal to $\Delta \Phi\left(i_{P}\right)=\min _{i} \Delta \Phi(i)$. Finally, the controller parameters are obtained using (34-35):

$$
T_{i}=\frac{\tau_{d}\left(i_{P}\right)}{\omega_{c M}^{*}} \tan \left(\frac{\pi}{180} \Delta \Phi\left(i_{P}\right)+\omega_{c M}^{*}\right)
$$

and

$$
k_{p}=\omega_{c M}^{*} \min \left\{\frac{A_{d}}{\tau_{d}}\right\} \sin \left(\frac{\pi}{180} \Delta \Phi\left(i_{P}\right)+\omega_{c M}^{*}\right)
$$




\section{Summary of Design Procedure}

The proposed tuning method is based on the above results about the influence of robustness margins on the time-domain performance of the controlled system. The different steps are as follows:

1. Compute the ID model parameters for the considered canal pool, for different discharges (e.g. $\left.\left[Q_{\min }, Q_{\max }\right]\right)$ and/or different friction coefficients.

2. Specify a reference perturbation scenario (i.e. a discharge $Q_{p}$ ), a maximum dimensional error $e_{\max }$, a maximal time $t_{10}$ to reach the reference level at $\pm 10 \%$ after this perturbation, and minimum gain and phase margins $\Delta G_{\min }$ and $\Delta \Phi_{\min }$.

3. Compute the lowest dimensionless maximum error $e_{\max }^{*}\left(i_{M}\right)$ and the corresponding gain margin $\Delta G_{M}$.

- If $\Delta G_{M}<\Delta G_{\min }$, the required performance is too high. The desired maximum error $e_{\max }$ needs to be increased.

- Else compute a first approximation of the proportional gain using Eq. (19).

4. Compute the dimensionless times $t_{10}^{*}(i)$ and the corresponding phase margins $\Delta \Phi(i)$. Compute the lowest phase margin $\Delta \Phi\left(i_{P}\right)$.

- If $\Delta \Phi\left(i_{P}\right)<\Delta \Phi_{\min }$, the required performance is too high. The time $t_{10}$ needs to be increased.

- Else compute the integral time $T_{i}$ and the proportional gain $k_{p}$ using Eqs. (23-24).

\section{Robust PI Controller Tuning for Pool 4 of ASCE Test Canal 1}

\section{Robust Controller Design}

Let us design a PI controllers using the above rules, with $e_{\max }=0.2 \mathrm{~m}$ and $t_{10}=3400 \mathrm{~s}$ for a unknown perturbation $Q_{p}=0.2 \mathrm{~m}^{3} / \mathrm{s}$. Following classical design requirements, a minimum gain margin of $\Delta G_{\min }=4 \mathrm{~dB}$ and a minimum phase margin of $\Delta \Phi_{\min }=30^{\circ}$ are required.

One can first check wether a stable controller exists that satisfies the design requirements. Indeed, with a minimum gain margin of $4 \mathrm{~dB}$, the dimensionless error is 1.48 , leading to a minimum dimensional error of $e_{\min }=1.48 \times y_{r}$, with $y_{r}$ the reference level, $y_{r}=Q_{p} \times \max \left\{\tau_{d} / A_{d}\right\}$. With the parameters of table 1, the reference level is found to be $y_{r}=0.10 \mathrm{~m}$. Then, the minimum error for the considered perturbation of $0.2 \mathrm{~m}^{3} / \mathrm{s}$ is equal to $e_{\min }=0.148 \mathrm{~m}$, which is smaller than $0.2 \mathrm{~m}$. Therefore there exists a stable PI controller with minimum margins that satisfies the design requirements. We choose to design a controller with the largest robustness margins, such that the design requirements are fulfilled.

The smallest dimensionless error computed using Eq. (38) is equal to $e_{\max }^{*}=2$, and the corresponding gain margin is therefore $\Delta G_{M}=10 \mathrm{~dB}$ (see table 7). The corresponding dimensionless crossover frequency is $\omega_{c M}^{*}=0.497$ [Eq.(32)].

A first approximation of the proportional gain is therefore $k_{p} \approx \min \left\{A_{d} / \tau_{d}\right\} \times \omega_{c M}^{*}=501 / 247 \times 0.497=$ $1.0 \mathrm{~m}^{2} / \mathrm{s}$.

With this approximation, one may compute the dimensionless crossover frequency and the gain margin for each model with Eqs. (20) and (21). Using the dimensionless minimum time, one may compute the corresponding phase margin with table 8 . These results are given in table 2. One observes that the worst case for $\Delta G$ corresponds to the high flow untuned situation, while the worst case for the phase margin corresponds to the low flow tuned situation.

[Table 2 about here.]

Finally, the controller parameters can be computed using Eqs. (23-24), leading to

$$
T_{i}=1457 \mathrm{~s}
$$

and

$$
k_{p}=0.91 \mathrm{~m}^{2} / \mathrm{s}
$$




\section{Simulation Results}

Fig. 2 shows the nonlinear simulations of the robust PI controller on the full Saint-Venant model for each of the four different hydraulic conditions. The scenario is an unpredicted perturbation of $Q_{p}=0.2$ $\mathrm{m}^{3} / \mathrm{s}$ occurring at time $600 \mathrm{~s}$. The simulations are done using SIC, a computer program developed by Cemagref, solving the complete nonlinear Saint-Venant equations with the semi-implicit Preissmann scheme (Malaterre and Baume, 1997).

The dotted lines on the figure represent the design specifications: the water level should not cross these lines to fulfill the design specification. One observes that the design specifications are fulfilled: the maximum error is smaller than $e_{\max }$ for each flow conditions and the time to reach $-0.1 \times y_{r}$ is smaller than $t_{10}$. The time responses are close to the linear ones (not displayed here for readability), and the design specifications are fulfilled. For this scenario, the nonlinear effects tend to decrease the maximum deviation from equilibrium compared to the linear case.

[Figure 2 about here.]

The robust design method of PI controllers for open-channel pool has therefore been validated for continuous-time control when the control action variable is the upstream discharge.

\section{Implementation Issues}

Let us now consider two important questions linked to the controller implementation: how do you take account of the sampling time and of the gates in the controller design?

\section{Sampled-Time Control}

Any applied controller is implemented digitally nowadays. This has to be taken into account in the controller design. An important issue is the fact that the sampled system may be very different from the continuous-time system if sampling is not performed properly. Therefore, the classical approach where one designs a continuous-time controller on the continuous-time system, and then passes the controller into discrete-time using a zero-order hold may not be suited. There are two different cases:

- If the sampling time $T_{s}$ can be chosen as desired, then we provide below a way to choose $T_{s}$ in order to recover the continuous-time performance. This usually leads to rather small $T_{s}$.

- Else, if $T_{s}$ is imposed, and is larger than the limit obtained above, the performance obtained with a continuous-time controller cannot be achieved. The sampling time cannot be neglected and it has to be included in the controller design. We propose to modify the continuous-time system in order to take sampling time into account.

The approach developed below assumes in both cases the use of an anti-aliasing filter $f(s)$, which is necessary for any sampled-time control system. $f(s)$ is either a low-pass analogous filter that is designed to cut frequencies higher than Nyquist frequency $\omega_{N}=\pi / T_{s}$, or a mechanical filter that averages the water level (e.g. a hydraulic filter). This filter is put before sampling, while the output of the controller is followed by a zero order hold $(\mathrm{ZOH})$ (see Fig. 3). The anti-aliasing filter ensures that noise will not influence the frequency response of the system. Before sampling, one should also ensure that the gain of the system is lower than 1 for frequencies higher than $\omega_{N} / 5$, i.e. for time periods smaller than $5 T_{s}$. This can be done by adequate scaling of measured variables.

[Figure 3 about here.]

Let us now consider both cases: how do you choose the sampling time when no constraints are imposed and how to take into account $T_{s}$ in the controller design when it is imposed? 


\section{Sampling Time Selection}

The robust PI tuning rules were developed for continuous-time controllers. However, for application purposes, one implements a discrete-time controller. If there are no constraints on the sampling period, the classical way is to design a continuous-time controller using the above tuning rules, and to sample it at a fast enough rate in order to recover the continuous-time performance. In that case, the sampling period can be selected according to the bandwidth of the controlled system, given by the dimensionless crossover frequency $\omega_{c}^{*}$.

A classical rule is that the Nyquist frequency $\omega_{N}=\pi / T_{s}$ should be 6 to 20 times larger than the crossover frequency (Aström and Wittenmark, 1990). Expressed in dimensionless form, this rule leads to:

$$
0.15 \leq T_{s}^{*} \omega_{c}^{*} \leq 0.5
$$

Using Eq. (32), this rule gives an interval for the sampling period of a canal controlled with a PI controller with a given gain margin (see table 3 ).

\section{[Table 3 about here.]}

One observes that for a canal pool controlled with a distant downstream PI controller with a gain margin of $10 \mathrm{~dB}$, the system should be sampled between 0.3 and 1 times the time delay in order to recover the continuous-time performance. This is consistent with the classical rules for time delay systems (Aström and Wittenmark, 1990, p. 233).

In the case of the PI controller designed for pool 4 of ASCE Test Canal one, the proposed rule would lead to: $74<T_{s}<247 \mathrm{~s}$. In the ASCE Test cases, the sampling time imposed is equal to $300 \mathrm{~s}$ for Test Canal one. Since this value is greater than the upper bound obtained from our developments, we cannot design a controller using a continuous-time approximation. It is necessary to take into account this constraint in the controller design.

\section{Imposed Sampling Time}

Let us now suppose that the dimensional sampling time $T_{s}$ is imposed on the canal.

The discrete-time controller transfer function is obtained by multiplying $K(s)$ by the transfer function of a zero order hold $F_{Z O H}(s)$, with:

$$
F_{Z O H}(s)=\frac{1-e^{-T_{s} s}}{T_{s} s}
$$

The frequency response of this transfer function is given by:

$$
\begin{aligned}
\left|F_{Z O H}(j \omega)\right| & =\frac{2 \sin \left(\frac{\omega T_{s}}{2}\right)}{\omega T_{s}} \\
\arg \left(F_{Z O H}(j \omega)\right) & =-\frac{\omega T_{s}}{2}
\end{aligned}
$$

with $j$ the complex number such that $j^{2}=-1$ and $\omega$ the frequency in $\mathrm{rad} / \mathrm{s}$.

One observes that a zero-order hold adds a supplementary delay of $T_{s} / 2$. Therefore, the controller can be tuned using the above rules, by considering a dimensional delay of $\tau_{d}+T_{s} / 2$ instead of $\tau_{d}$. The modification induced by the zero-order hold on the gain of the system can be taken into account by specifying a modified gain margin $\tilde{\Delta} G$ :

$$
\tilde{\Delta} G=\Delta G-20 \log _{10}\left|F_{Z O H}\left(j \omega_{180}\right)\right|
$$

in order to obtain the desired gain margin $\Delta G$ on the sampled-time system.

Therefore, an imposed sampling time will add a constraint on the achievable performance, since it increases the time-delay of the system. However, with the proposed rule, one can design a robust controller using exactly the same method as proposed above. 


\section{Conversion from Discharge to Gate Opening}

The PI controllers presented above use as control variable the upstream discharge. In practice, since canals are controlled with gates, one needs to compute the corresponding gate opening to obtain a desired discharge.

One may think that the solution to this problem is obvious: if the gate upstream and downstream water elevations are measured, it seems sufficient to invert the gate discharge equation in order to compute the required gate opening to let a desired discharge flow through the gate. However, this is only true if one is able to measure the gate upstream and downstream water levels and to operate the gate continuously along time. For computer controlled system, this is not possible, since the controller operates at a given sampling time step. There are also mechanical constraints on the gates' motors, which cannot be operated continuously. And finally, the downstream gate water elevation is not always measured.

This problem is therefore not as simple as it may seem at first sight. For lack of space, we will only consider a simple solution to this problem, but other solutions are possible (see Malaterre and Baume (1999)).

The considered canal pool controlled with the upstream gate opening is depicted in Fig. 4 .

[Figure 4 about here.]

Let us denote the upstream gate discharge equation

$$
Q=C_{d} L_{v} W \sqrt{2 g\left(Y_{1}-Y_{2}\right)}
$$

with $Y_{1}$ the gate upstream water level, $Y_{2}$ the gate downstream water level, and $W$ the gate opening. Since we consider small deviations from equilibrium values, the linearized upstream gate equation is

$$
q=k_{1} y_{1}+k_{2} y_{2}+k_{w} w
$$

with $k_{1}=Q /\left(2\left(Y_{1}-Y_{2}\right)\right), k_{2}=-k_{1}$ and $k_{w}=Q / W$.

In this equation, only the term $k_{2} y_{2}$ introduces a local feedback which modifies the dynamics of the ID model. Nevertheless, the coupling between the downstream pool water level and the upstream pool water level is generally very low for partially submerged gates, which leads to neglect this term. In this way, the PI tuning rules can be applied on the following system:

$$
y=\frac{k_{w}}{A_{d} s} e^{-\tau_{d} s} w-\frac{1}{A_{d} s}\left(p+k_{1} e^{-\tau_{d} s} y_{1}\right)
$$

The term $k_{1} y_{1}$ appears as a perturbation in the upstream discharge, and corresponds to the hydraulic coupling between successive canal pools, for case where the upstream gate water level is not constant. It acts in the same way as a downstream discharge perturbation.

This simple approximation provides a good representation of the canal pool controlled by a gate in the frequency range of interest, and generally leads to an efficient PI controller. A more technical justification of this point can be provided, but is omitted here for lack of space.

Finally, the proposed PI tuning method can be applied on a canal pool controlled with a gate, but now the proportional gain $k_{p}$ needs to be divided by $k_{w}$. For a robust tuning, the tuning method can be used by replacing $A_{d}$ by $A_{d} / k_{w}$ and $Q_{p}$ by $\max \left|Q_{p} / k_{w}\right|$ in the controller design formulas. This corresponds to the largest perturbation, which will lead to the largest error. Therefore, if the design requirements are fulfilled for this perturbation, then they will be fulfilled for smaller perturbations.

\section{Application to Pool 4 of ASCE Test Canal 1}

Let us illustrate the approach on pool 4 of ASCE Test Canal 1, by designing a controller acting on the upstream gate, with a pre-specified sampling time of $T_{s}=300 \mathrm{~s}$.

The delay needs to be changed from $\tau_{d}$ to $\tau_{d}+T_{s} / 2$. The gate coefficient $k_{w}$ is also computed for each model. Results are displayed in table 4 .

[Table 4 about here.] 
With a sampling period of $300 \mathrm{~s}$, it is not possible to guarantee a maximum error of $0.2 \mathrm{~m}$ for a perturbation of $0.2 \mathrm{~m}^{3} / \mathrm{s}$. Indeed, with a minimum gain margin of $4 \mathrm{~dB}$, the dimensionless error is 1.48 , leading to a minimum dimensional error of $e_{\min }=1.48 \times y_{r}$. The reference level is obtained by $y_{r}=Q_{p} \times \max \left\{\left(\tau_{d}+T_{s} / 2\right) / A_{d}\right\}=0.158 \mathrm{~m}$ with the parameters of table 4 . Then, the minimum error for the considered perturbation of $0.2 \mathrm{~m}^{3} / \mathrm{s}$ is equal to

$$
e_{\min }=1.48 \times 0.158=0.23 \mathrm{~m}
$$

Let us specify a maximum error of $0.25 \mathrm{~m}$, and a time $t_{10}=4000 \mathrm{~s}$. The smallest dimensionless error computed using Eq. (38) is equal to 1.57, and the corresponding gain margin is therefore $\Delta G_{M}=5 \mathrm{~dB}$. The corresponding dimensionless crossover frequency is $\omega_{c M}^{*}=0.88$.

A first approximation of the proportional gain is therefore $k_{p}=\min \left\{A_{d} / k_{w} /\left(\tau_{d}+T_{s} / 2\right)\right\} \times \omega_{c M}^{*}=0.276$. Following the same line as above, one gets the final controller parameters

$$
k_{p}=0.27 \mathrm{~m} / \mathrm{m}
$$

and

$$
T_{i}=2067 \mathrm{~s}
$$

Simulation results on a full nonlinear model including the upstream gate are depicted in Fig. 5. Once again, the results are close to the linear simulation (not presented here). The maximum deviation from equilibrium is smaller than the required value $0.25 \mathrm{~m}$, and the settling time condition is also fulfilled.

[Figure 5 about here.]

\section{Application to Pool 8 of ASCE Test Canal 2}

In order to illustrate the method on a different canal pool, we chose pool 8 of ASCE Test Canal 2, which is $2 \mathrm{~km}$ long, with bottom width $5 \mathrm{~m}$ and bed slope 0.0001 . The downstream target water level is $1.7 \mathrm{~m}$. The Manning coefficient is 0.02 for tuned conditions, and 0.026 for untuned conditions. The sampling time is $T_{s}=900 \mathrm{~s}$. The model parameters are given in table 5 .

[Table 5 about here.]

The maximum perturbation is $4 \mathrm{~m}^{3} / \mathrm{s}$. The reference level is obtained by $y_{r}=Q_{p} \times \max \left\{\left(\tau_{d}+T_{s} / 2\right) / A_{d}\right\}=$ $0.23 \mathrm{~m}$ with the parameters of table 5 .

With a minimum gain margin of $4 \mathrm{~dB}$, the dimensionless error is 1.48 , leading to a minimum dimensional error of $e_{\min }=1.48 \times y_{r}$.

Then, the minimum error for the considered perturbation of $4 \mathrm{~m}^{3} / \mathrm{s}$ is equal to

$$
e_{\min }=1.48 \times 0.23=0.34 \mathrm{~m}
$$

In order to show another type of application of the method, we choose to design a PI controller with a given gain margin of $6 \mathrm{~dB}$, and a phase margin ratio of 0.5 . The corresponding dimensionless crossover frequency is $\omega_{c M}^{*}=0.787$.

A first approximation of the proportional gain is therefore $k_{p}=\min \left\{A_{d} / k_{w} /\left(\tau_{d}+T_{s} / 2\right)\right\} \times \omega_{c M}^{*}=$ $1.11 \times 0.787=0.87$.

With this approximation, one may compute the crossover frequency and the gain margin for each model with Eqs. (20) and (21). Indeed, the approximate crossover frequencies are given for each case by:

$$
\omega_{c}=k_{p}\left(\tau_{d}+T_{s} / 2\right) \frac{k_{w}}{A_{d}}
$$

Using the phase margin ratio of 0.5 , one may compute the corresponding phase margin from the maximum phase margin. These results are given in table 6 .

[Table 6 about here.] 
One then gets the final controller parameters $k_{p}=0.81 \mathrm{~m} / \mathrm{m}$ and $T_{i}=3125 \mathrm{~s}$.

Simulation results on a full nonlinear model including the upstream gate are depicted in Figs. 6 and 7. For high flow conditions, the total perturbation is equal to $4 \mathrm{~m}^{3} / \mathrm{s}$, while for low flow conditions, we used a perturbation of $0.4 \mathrm{~m}^{3} / \mathrm{s}$. It is clear from the figures that the behavior is qualitatively the same for both situations. The oscillating modes can be seen in the response, because they are not controlled. However, the overall shape of the response is similar to the one obtained with a ID model.

One observes that the maximum deviation from equilibrium is about $0.3 \mathrm{~m}$ in the high flow case, while it is $0.03 \mathrm{~m}$ in the low flow case. This is consistent with the predicted values obtained with a linear ID model. Indeed, in the low flow case, using the gain margin of table 6 and the parameters of table 5, one gets a maximum deviation of about $0.033 \mathrm{~m}$ for a perturbation of $0.4 \mathrm{~m}^{3} / \mathrm{s}$ in tuned and untuned conditions. In the high flow case, the linear computations are more far away from the nonlinear results, since the maximum deviations are $0.52 \mathrm{~m}$ and $0.58 \mathrm{~m}$ in the tuned and untuned conditions. However, in this case, the linear approximation is no longer valid, since the perturbation of $4 \mathrm{~m}^{3} / \mathrm{s}$ is of the same order as the initial flow of $5 \mathrm{~m}^{3} / \mathrm{s}$. To get a better estimate, one could use the ID model parameters corresponding to the final state, where the flow is $1 \mathrm{~m}^{3} / \mathrm{s}$. In this case, the maximum deviations are about $0.33 \mathrm{~m}$ in both cases, which is much closer to nonlinear simulations results.

[Figure 6 about here.]

[Figure 7 about here.]

\section{Conclusion}

Based on the results of the companion paper, this paper develops a PI controller tuning method to ensure robust performance: this means that the controller ensures a minimum performance level for a set of hydraulic conditions. The performance requirements include the maximum error after an unpredicted perturbation has occurred and the time to reach the design level after this. The results are first derived for continuous-time control, then extended to discrete-time control. The control action variable can be the upstream discharge or the gate opening. This robust tuning method enables us to :

1. Evaluate whether a required performance is possible, and if it is the case,

2. To compute the controller parameters that fulfills the design requirements for all considered hydraulic conditions.

The proposed tuning methodology appears to be flexible and simple enough to be appealing to irrigation canal managers in order to tune distant downstream PI controllers for canal pools. It has been applied in simulation on two different canal pools taken from the ASCE test cases. The results are very satisfactory, since the simulations on a full nonlinear model are consistent with the results obtained from linear simulations. This method is shown to lead to efficient realistic PI controllers for a canal pool.

A natural extension of this work concerning the design of controllers for multiple pools irrigation canals is the subject of current research. The proposed tuning method is implemented in SIC, a computer program developed by Cemagref for modelling and control of irrigation canals.

Finally, combined with the computation of ID model parameters from physical parameters (following e.g. Litrico and Fromion (2004)), the proposed tuning method enables us to directly link the achievable performance of a controlled canal pool to its physical parameters. This feature could be used to take account of the control objectives in the structural design of a new canal.

\section{Acknowledgments}

The authors acknowledge the financial help of Cemagref and INRA through the collaborative program ASS AQUAE $n^{\circ} 2$. 


\section{Notations}

The following symbols are used in this paper:

* = superscript for dimensionless variables;

$\sim$ notation for modified variables;

$A_{d}=$ downstream backwater area in $\mathrm{m}^{2}$;

$C_{d}=$ discharge coefficient;

$e_{\max }=$ maximum water level deviation in response to a discharge perturbation;

$F_{Z O H}=$ Zero Order Hold transfer function;

$f(s)=$ anti-aliasing filter;

$G_{i}(s)=$ transfer functions;

$j=$ complex number $j^{2}=-1$;

$K_{1}=$ controller transfer function;

$k_{p}=$ proportional gain in $\mathrm{m}^{2} / \mathrm{s}$ or in $\mathrm{m} / \mathrm{m}$;

$k_{1}, k_{2}, k_{w}=$ proportional constants;

$i=$ index of models;

$i_{M}=$ index of model leading to the lowest dimensionless error;

$i_{P}=$ index of model leading to the lowest settling time;

$N=$ number of models;

$p=$ downstream perturbation in $\mathrm{m}^{3} / \mathrm{s}$;

$Q=$ absolute discharge in $\mathrm{m}^{3} / \mathrm{s}$;

$Q_{p}=$ perturbation discharge in $\mathrm{m}^{3} / \mathrm{s}$;

$q=$ relative discharge in $\mathrm{m}^{3} / \mathrm{s}$;

$s=$ Laplace variable in $\mathrm{s}^{-1}$;

$T_{i}=$ integral time in $\mathrm{s}$;

$T_{s}=$ sampling time period in $\mathrm{s} ;$

$t_{10}=$ settling time after a step perturbation in $\mathrm{s}$;

$u_{1}=$ deviation in upstream control variable in $\mathrm{m}^{3} / \mathrm{s}$;

$u_{2}=$ deviation in downstream control variable in $\mathrm{m}^{3} / \mathrm{s}$;

$W=$ absolute gate opening in $\mathrm{m}$;

$w=$ relative gate opening in $\mathrm{m}$;

$Y_{1}=$ gate upstream water level in $\mathrm{m}$;

$Y_{1}=$ gate downstream water level in $\mathrm{m}$;

$y=$ deviation in water level in $\mathrm{m}$;

$y_{r}=$ reference water level deviation in $\mathrm{m}$;

$\Delta G=$ gain margin in $\mathrm{dB}$;

$\Delta G_{M}=$ gain margin for the minimum error specification in $\mathrm{dB}$;

$\Delta G_{\min }=$ minimum gain margin in $\mathrm{dB}$;

$\Delta \Phi=$ phase margin in degrees;

$\Delta \Phi_{\min }=$ minimum phase margin in degrees;

$\Delta \Phi_{\max }=$ maximum phase margin in degrees;

$\omega=$ frequency in $\mathrm{rad} / \mathrm{s}$;

$\omega_{180}=$ frequency at $-180^{\circ}$ in $\mathrm{rad} / \mathrm{s}$;

$\omega_{c}=$ crossover frequency in $\mathrm{rad} / \mathrm{s}$;

$\omega_{c M}=$ crossover frequency for gain margin $\Delta G_{M}$ in $\mathrm{rad} / \mathrm{s}$;

$\tau_{d}=$ time-delay for downstream propagation in s;

\section{Appendix I. Nominal PI Controller Design}

Having specified desired robustness margins $(\Delta G, \Delta \Phi)$ according to desired time-domain performance specifications one may compute the PI controller parameters according to the following formulas. The 
ID model is first put in dimensionless form, by defining $s^{*}=\tau_{d} s$ the dimensionless Laplace variable, $y_{r}=\tau_{d} Q_{p} / A_{d}$ the reference water level deviation, $y^{*}=y / y_{r}$ the dimensionless water level, $u_{1}^{*}=u_{1} / Q_{p}$ the dimensionless upstream discharge, and $p^{*}=p / Q_{p}$ the dimensionless water withdrawal. $Q_{p}$ is the reference discharge perturbation.

First, the dimensionless frequency $\omega_{180}^{*}$ is given by:

$$
\omega_{180}^{*}=\frac{\pi}{2}
$$

Then, with the chosen gain margin $\Delta G$ (expressed in $\mathrm{dB}$ ), the dimensionless crossover frequency is obtained by:

$$
\omega_{c}^{*}=\omega_{180}^{*} 10^{-\frac{\Delta G}{20}}
$$

Then, if the chosen phase margin $\Delta \Phi$ is lower than the maximum phase margin

$$
\Delta \Phi_{\max }=90\left(1-10^{-\frac{\Delta G}{20}}\right)
$$

the dimensionless controller parameters are obtained by:

$$
T_{i}^{*}=\frac{1}{\omega_{c}^{*}} \tan \left(\frac{\pi}{180} \Delta \Phi+\omega_{c}^{*}\right)
$$

and

$$
k_{p}^{*}=\frac{T_{i}^{*} \omega_{c}^{* 2}}{\sqrt{1+T_{i}^{* 2} \omega_{c}^{* 2}}}
$$

The dimensional PI controller parameters can be obtained from the dimensionless parameters by the equations:

$$
k_{p}=k_{p}^{*} \frac{A_{d}}{\tau_{d}}
$$

and

$$
T_{i}=\tau_{d} T_{i}^{*}
$$

\section{Appendix II. Time-Domain Performance Related to Robustness Margins}

The companion paper's results on the relation between time-domain performance and robustness margins can be summarized with the following two tables. Table 7 gives the relation between the gain margin and the dimensionless maximum deviation from equilibrium corresponding to a unit downstream perturbation.

To a controller with a given gain margin corresponds a maximum dimensionless deviation from equilibrium $e_{\max }^{*}$. The dimensional maximum deviation from equilibrium for the downstream water level corresponding to a withdrawal of $Q_{p}$ can then be computed using the following equation:

$$
e_{\max }=\frac{e_{\max }^{*} \tau_{d} Q_{p}}{A_{d}}
$$

[Table 7 about here.]

Table 8 gives the dimensionless time $t_{10}^{*}$ to reach $0.1 \times y_{r}$ after such a perturbation has occurred. Tables 7 and 8 are very useful to translate time-domain requirements into robustness margins constraints.

[Table 8 about here.] 


\section{References}

Aström, K. and Wittenmark, B. (1990). Computer controlled systems: theory and design. Prentice-Hall International Editions, London. 544 p.

Baume, J.-P., Malaterre, P.-O., and Sau, J. (1999). Tuning of PI to control an irrigation canal using optimization tools. In Workshop on Modernization of Irrigation Water Delivery Systems, pages 483500, Phoenix, Arizona, USA.

Clemmens, A. J., Kacerek, T. F., Grawitz, B., and Schuurmans, W. (1998). Test cases for canal control algorithms. J. Irrig. Drain. Eng., 124(1):23-30.

Clemmens, A. J. and Schuurmans, J. (2004). Simple optimal downstream feedback canal controllers: theory. J. Irrig. Drain. Eng., 130(1):26-34.

Clemmens, A. J. and Wahlin, B. (2004). Simple optimal downstream feedback canal controllers: ASCE test case results. J. Irrig. Drain. Eng., 130(1):35-46.

Deltour, J.-L. and Sanfilippo, F. (1998). Introduction of Smith predictor into dynamic regulation. J. Irrig. Drain. Eng., 124(4):47-52.

Litrico, X. and Fromion, V. (2004). Simplified modelling of irrigation canals for controller design. $J$. Irrig. and Drain. Engrg., 130(5):373-383.

Malaterre, P.-O. and Baume, J.-P. (1997). SIC 3.0, a simulation model for canal automation design. In Int. Workshop on the Regulation of Irrigation Canals RIC'97, Marrakech (Morocco).

Malaterre, P.-O. and Baume, J.-P. (1999). Optimum choice of control action variables and linked algorithms. comparison of different alternatives. In Workshop on Modernization of Irrigation Water Delivery Systems, pages 387-406, Phoenix, Arizona, USA.

Schuurmans, J. (1997). Control of water levels in open-channels. Ph.D. thesis, ISBN 90-9010995-1, Delft University of Technology.

Schuurmans, J., Clemmens, A. J., Dijkstra, S., Hof, A., and Brouwer, R. (1999a). Modeling of irrigation and drainage canals for controller design. J. Irrig. Drain. Eng., 125(6):338-344.

Schuurmans, J., Hof, A., Dijkstra, S., Bosgra, O. H., and Brouwer, R. (1999b). Simple water level controller for irrigation and drainage canals. J. Irrig. Drain. Eng., 125(4):189-195. 


\section{List of Figures}

1 Linear ID model simulation of the distant downstream PI controller to a downstream perturbation of $0.2 \mathrm{~m}^{3} / \mathrm{s}$, high and low flow, tuned and untuned conditions, pool 4 of ASCE test canal $1 \ldots \ldots \ldots \ldots \ldots \ldots \ldots$

2 Nonlinear Saint-Venant model simulation of the continuous time distant downstream PI controller to a downstream perturbation of $0.2 \mathrm{~m}^{3} / \mathrm{s}$, high and low flow, tuned and untuned conditions, pool 4 of ASCE test canal $1 \ldots \ldots \ldots \ldots$

3 Sampled-time control . . . . . . . . . . . . . . . . . . . . . . . . . . . . . . 19

4 Schematic representation of a canal pool controlled with the upstream gate . . . . . . 20

5 Nonlinear Saint-Venant model simulation of the discrete-time distant downstream PI controller to a downstream perturbation of $0.2 \mathrm{~m}^{3} / \mathrm{s}$, high and low flow, tuned and untuned conditions, pool 4 of ASCE test canal $1 \ldots \ldots \ldots \ldots$ troller to a downstream perturbation of $4 \mathrm{~m}^{3} / \mathrm{s}$, high flow, tuned and untuned conditions, pool 8 of ASCE test canal $2 \ldots \ldots \ldots \ldots \ldots$

7 Nonlinear Saint-Venant model simulation of the discrete-time distant downstream PI controller to a downstream perturbation of $0.4 \mathrm{~m}^{3} / \mathrm{s}$, low flow, tuned and untuned conditions, pool 8 of ASCE test canal $2 \ldots \ldots \ldots \ldots \ldots \ldots$ 

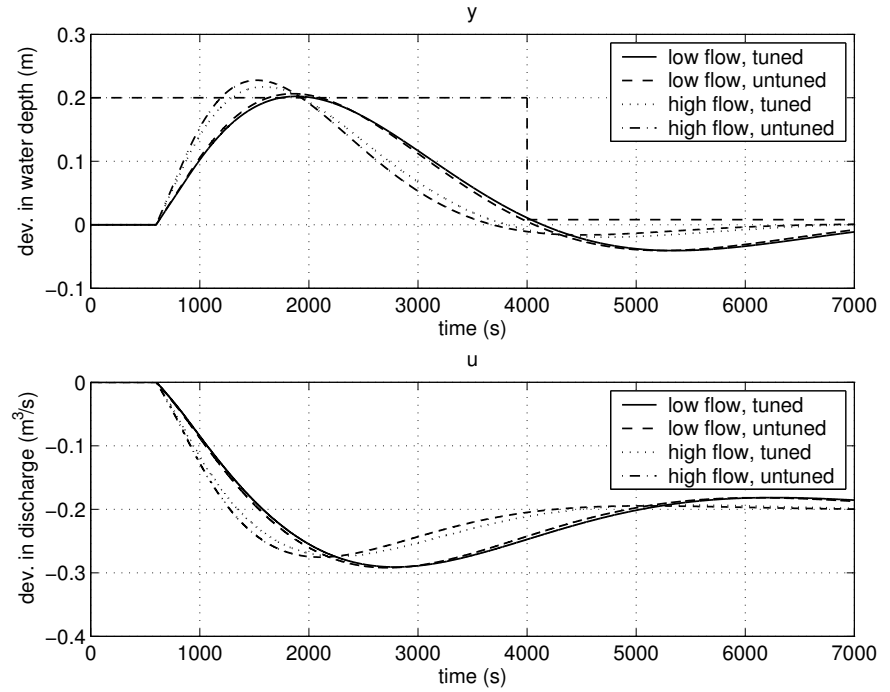

Figure 1: Linear ID model simulation of the distant downstream PI controller to a downstream perturbation of $0.2 \mathrm{~m}^{3} / \mathrm{s}$, high and low flow, tuned and untuned conditions, pool 4 of ASCE test canal 1 

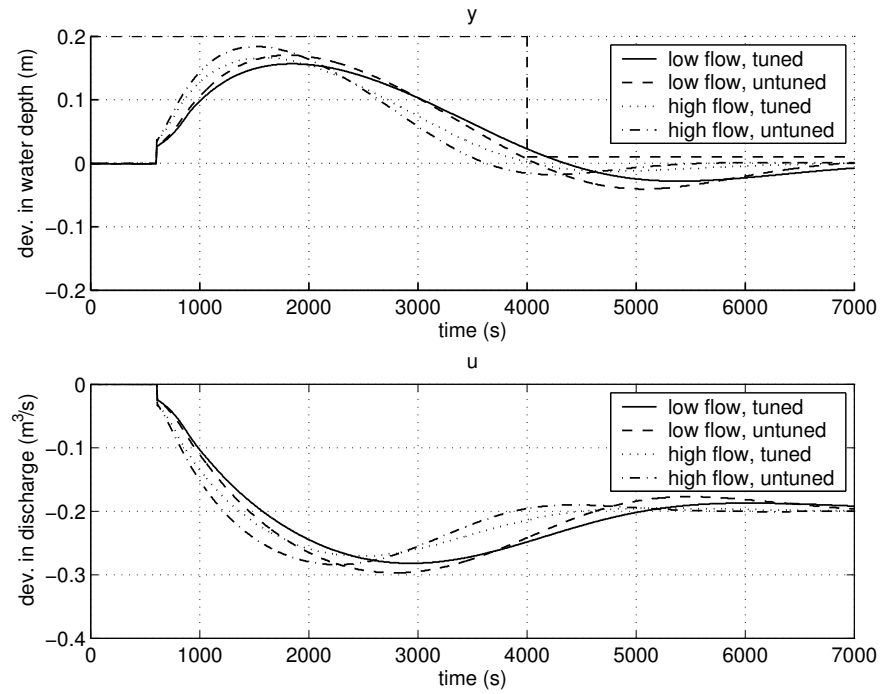

Figure 2: Nonlinear Saint-Venant model simulation of the continuous time distant downstream PI controller to a downstream perturbation of $0.2 \mathrm{~m}^{3} / \mathrm{s}$, high and low flow, tuned and untuned conditions, pool 4 of ASCE test canal 1 


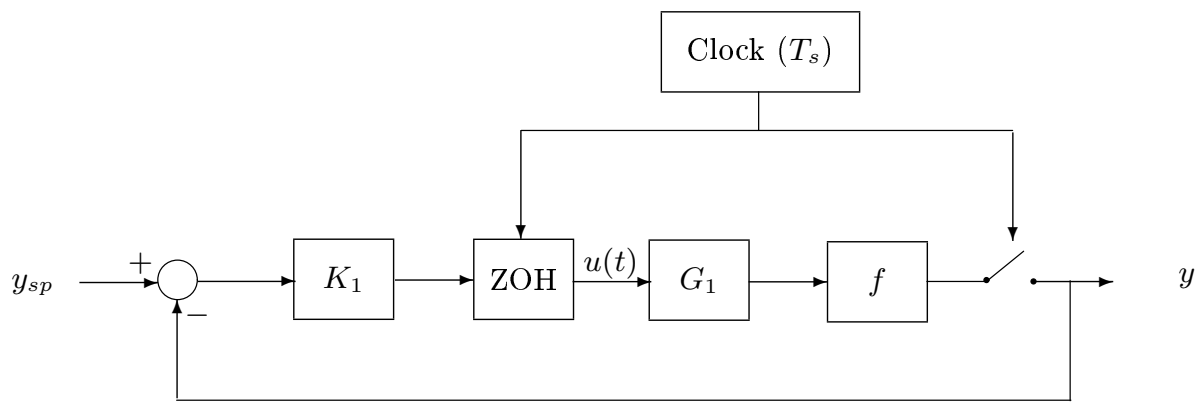

Figure 3: Sampled-time control 


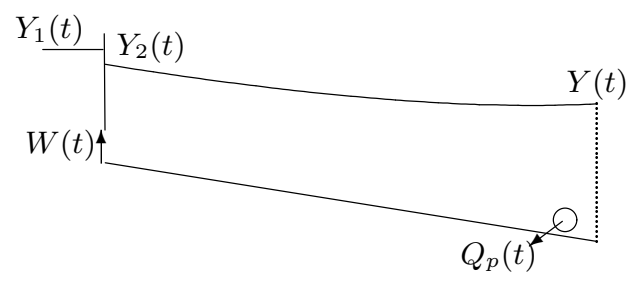

Figure 4: Schematic representation of a canal pool controlled with the upstream gate 

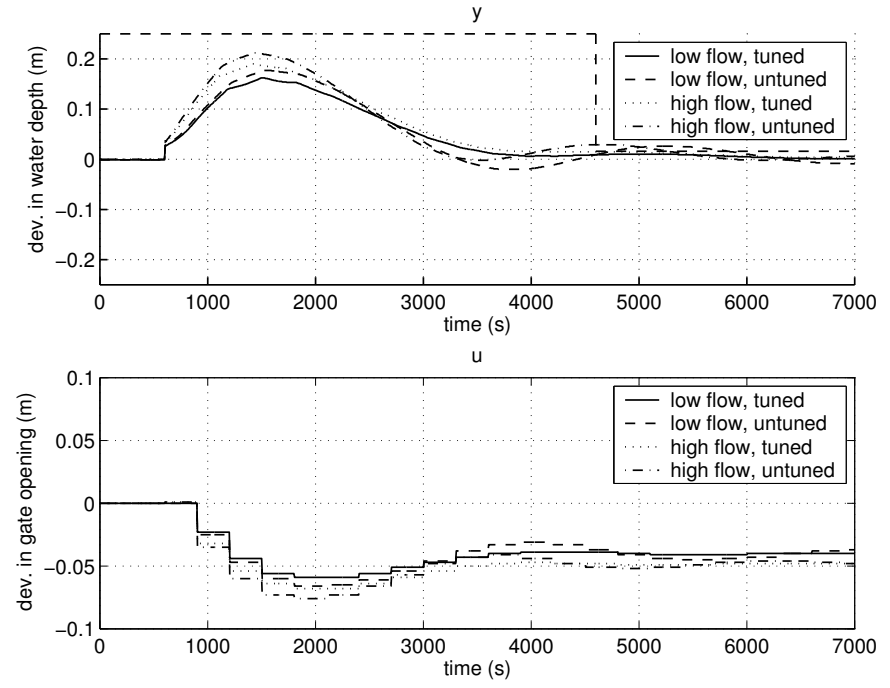

Figure 5: Nonlinear Saint-Venant model simulation of the discrete-time distant downstream PI controller to a downstream perturbation of $0.2 \mathrm{~m}^{3} / \mathrm{s}$, high and low flow, tuned and untuned conditions, pool 4 of ASCE test canal 1 

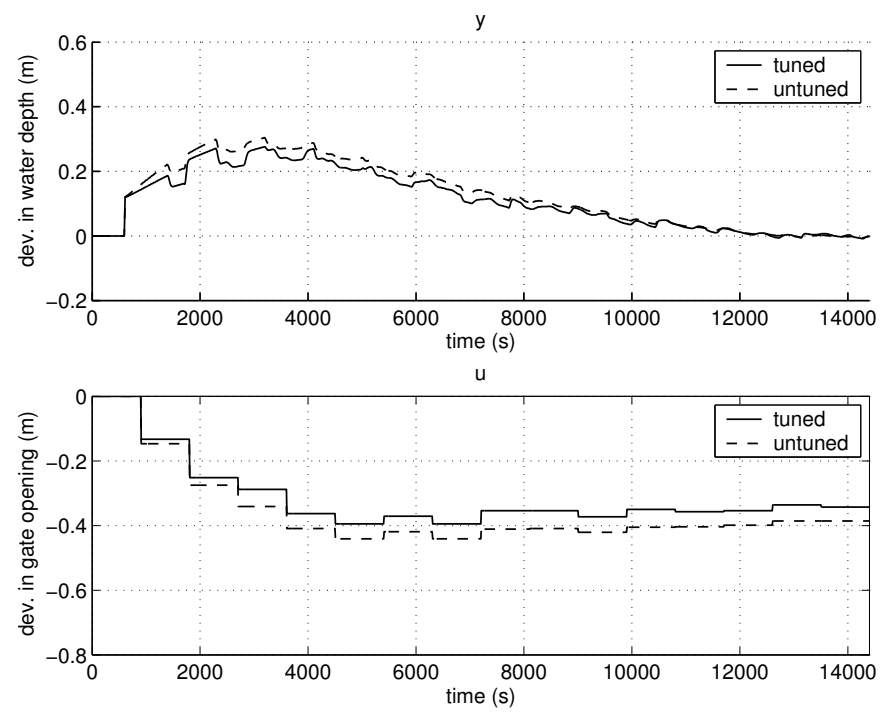

Figure 6: Nonlinear Saint-Venant model simulation of the discrete-time distant downstream PI controller to a downstream perturbation of $4 \mathrm{~m}^{3} / \mathrm{s}$, high flow, tuned and untuned conditions, pool 8 of ASCE test canal 2 

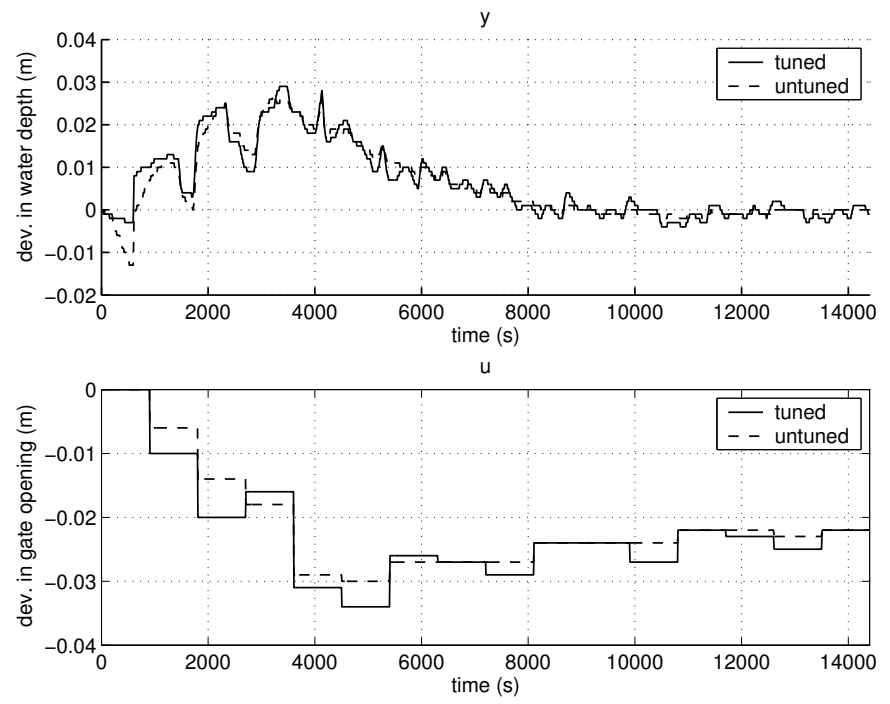

Figure 7: Nonlinear Saint-Venant model simulation of the discrete-time distant downstream PI controller to a downstream perturbation of $0.4 \mathrm{~m}^{3} / \mathrm{s}$, low flow, tuned and untuned conditions, pool 8 of ASCE test canal 2 


\section{List of Tables}

1 ID model parameters of Pool 4 of ASCE Test Canal 1 for different hydraulic conditions 25

2 Dimensionless crossover frequency, gain, and phase margins for different hydraulic conditions 26

3 Minimum and maximum dimensionless sampling time $T_{s}^{*}$ for different gain margins $\Delta G \quad 27$

4 ID model parameters of Pool 4 of ASCE Test Canal 1 for different hydraulic conditions 28

5 ID model parameters of Pool 8 of ASCE Test Canal 2 for different hydraulic conditions 29

6 Dimensionless crossover frequency, gain and phase margins for different hydraulic conditions 30

7 Lower bound on $e_{\max }^{*}$ for different gain margins $\Delta G \ldots \ldots \ldots \ldots$

8 Dimensionless time $t_{10}^{*}$ to reach $0.1 \times y_{r}$ after a step perturbation as function of $\Delta G$ and

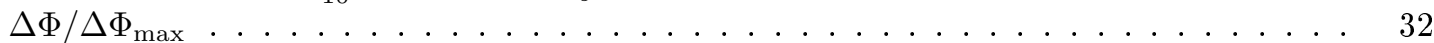


Table 1: ID model parameters of Pool 4 of ASCE Test Canal 1 for different hydraulic conditions

\begin{tabular}{c|c|ccc}
\hline \hline$Q\left(\mathrm{~m}^{3} / \mathrm{s}\right)$ & situation & $\tau_{d}(\mathrm{~s})$ & $A_{d}\left(\mathrm{~m}^{2}\right)$ & $A_{d} / \tau_{d}\left(\mathrm{~m}^{2} / \mathrm{s}\right)$ \\
\hline 0.5 & tuned & 308 & 775 & 2.516 \\
0.5 & untuned & 315 & 752 & 2.387 \\
1.4 & tuned & 238 & 546 & 2.294 \\
1.4 & untuned & 247 & 501 & 2.028 \\
\hline
\end{tabular}


Table 2: Dimensionless crossover frequency, gain, and phase margins for different hydraulic conditions

\begin{tabular}{c|cccc}
\hline \hline$Q\left(\mathrm{~m}^{3} / \mathrm{s}\right)$ & situation & $\omega_{c}^{*}$ & $\Delta G(\mathrm{~dB})$ & $\Delta \Phi(\mathrm{deg})$ \\
\hline 0.5 & tuned & 0.397 & 11.9 & 38.3 \\
0.5 & untuned & 0.419 & 11.5 & 39.0 \\
1.4 & tuned & 0.438 & 11.1 & 44.2 \\
1.4 & untuned & 0.496 & 10.0 & 43.1 \\
\hline \hline
\end{tabular}


Table 3: Minimum and maximum dimensionless sampling time $T_{s}^{*}$ for different gain margins $\Delta G$

\begin{tabular}{c|cccccccccc}
\hline \hline$\Delta G(\mathrm{~dB})$ & 6 & 7 & 8 & 9 & 10 & 11 & 12 & 13 & 14 & 15 \\
\hline$T_{s}^{*} \min$. & 0.19 & 0.21 & 0.24 & 0.27 & 0.30 & 0.34 & 0.38 & 0.43 & 0.48 & 0.54 \\
$T_{s}^{*} \max$. & 0.64 & 0.71 & 0.80 & 0.90 & 1.0 & 1.13 & 1.27 & 1.42 & 1.60 & 1.80 \\
\hline \hline
\end{tabular}


Table 4: ID model parameters of Pool 4 of ASCE Test Canal 1 for different hydraulic conditions

\begin{tabular}{c|c|ccc}
\hline \hline$Q\left(\mathrm{~m}^{3} / \mathrm{s}\right)$ & situation & $\tau_{d}+T_{s} / 2(\mathrm{~s})$ & $A_{d}\left(\mathrm{~m}^{2}\right)$ & $k_{w}\left(\mathrm{~m}^{2} / \mathrm{s}\right)$ \\
\hline 0.5 & tuned & 458 & 775 & 4.98 \\
0.5 & untuned & 465 & 752 & 4.94 \\
1.4 & tuned & 388 & 546 & 4.19 \\
1.4 & untuned & 397 & 501 & 4.02 \\
\hline
\end{tabular}


Table 5: ID model parameters of Pool 8 of ASCE Test Canal 2 for different hydraulic conditions

\begin{tabular}{c|c|ccc}
\hline \hline$Q\left(\mathrm{~m}^{3} / \mathrm{s}\right)$ & situation & $\tau_{d}+T_{s} / 2(\mathrm{~s})$ & $A_{d}\left(\mathrm{~m}^{2}\right)$ & $k_{w}\left(\mathrm{~m}^{2} / \mathrm{s}\right)$ \\
\hline 1 & tuned & 1017 & 19523 & 13.39 \\
1 & untuned & 1017 & 19469 & 9.51 \\
\hline 5 & tuned & 964 & 17806 & 7.77 \\
5 & untuned & 961 & 16738 & 6.91 \\
\hline \hline
\end{tabular}


Table 6: Dimensionless crossover frequency, gain and phase margins for different hydraulic conditions

\begin{tabular}{c|cccc}
\hline \hline$Q\left(\mathrm{~m}^{3} / \mathrm{s}\right)$ & situation & $\omega_{c}^{*}$ & $\Delta G(\mathrm{~dB})$ & $\Delta \Phi(\mathrm{deg})$ \\
\hline 1 & tuned & 0.777 & 6.1 & 22.8 \\
1 & untuned & 0.787 & 6.0 & 22.5 \\
5 & tuned & 0.368 & 12.6 & 34.4 \\
5 & untuned & 0.348 & 13.1 & 35.0 \\
\hline \hline
\end{tabular}


Table 7: Lower bound on $e_{\max }^{*}$ for different gain margins $\Delta G$

\begin{tabular}{c|ccccccccc}
\hline \hline$\Delta G(\mathrm{~dB})$ & 1 & 2 & 4 & 6 & 8 & 10 & 12 & 14 & 16 \\
\hline$e_{\max }^{*}$ & 1.35 & 1.39 & 1.48 & 1.61 & 1.77 & 2 & 2.3 & 2.72 & 3.26 \\
\hline \hline
\end{tabular}


Table 8: Dimensionless time $t_{10}^{*}$ to reach $0.1 \times y_{r}$ after a step perturbation as function of $\Delta G$ and $\Delta \Phi / \Delta \Phi_{\max }$

\begin{tabular}{c|ccccccccc}
\hline \hline & \multicolumn{10}{|c}{$\Delta G(\mathrm{~dB})$} \\
\hline$\Delta \Phi / \Delta \Phi_{\max }$ & 1 & 2 & 4 & 6 & 8 & 10 & 12 & 14 & 16 \\
\hline 0.1 & 3.1 & 3.15 & 3.55 & 4.2 & 5.15 & 6.4 & 8.0 & 10.05 & 12.65 \\
0.2 & 3.15 & 3.2 & 3.6 & 4.25 & 5.2 & 6.5 & 8.15 & 10.25 & 12.9 \\
0.3 & 3.16 & 3.25 & 3.65 & 4.35 & 5.35 & 6.70 & 8.45 & 10.65 & 13.45 \\
0.4 & 3.20 & 3.30 & 3.75 & 4.50 & 5.60 & 7.05 & 8.95 & 11.35 & 14.35 \\
0.5 & 3.25 & 3.40 & 3.90 & 4.75 & 6.0 & 7.70 & 9.80 & 12.50 & 15.9 \\
0.6 & 3.30 & 3.50 & 4.10 & 5.20 & 6.95 & 9.05 & 11.55 & 14.70 & 18.65 \\
0.7 & 3.40 & 3.65 & 4.70 & 11.15 & 12.40 & 13.60 & 16.15 & 19.90 & 24.85 \\
0.8 & 3.50 & 4.0 & 18.60 & 18.60 & 20.65 & 24.15 & 29.05 & 35.55 & 44.0 \\
0.9 & 3.70 & 50.60 & 38.85 & 39.40 & 44.45 & 52.90 & 64.95 & 81.25 & 150.0 \\
\hline \hline
\end{tabular}

